\title{
Radial Diffusion in Jupiter's Magnetosphere
}

\author{
D. N. BAKER ${ }^{1}$ AND C. K. Goertz \\ Department of Physics and Astronomy, University of Iowa, lowa City, lowa 52242
}

\begin{abstract}
Radial phase space density profiles for equatorially mırroring particles are computed from the data of the University of lowa packages on Pioneer 10 . The profiles are only consistent with radial diffusion subject to nonadiabatic losses. It is suggested that these losses are due to pitch angle scattering by whistler turbulence.
\end{abstract}

\section{INTRODUCTION}

Before the Pioneer 10 and 11 Jupiter flybys, it was suggested that the Jovian radiation belts are populated by strong radial inward diffusion [see, e.g., Birmingham et al., 1974; Coroniti. $1974 ;$ Mead, 1972] violating the third adiabatic invariant. Birmingham et al. [1974] and Coroniti [1974] showed that the general features of the Jovian decimetric radiation (DIM) can be explained in terms of a diffusion solution for the energetic particles. Mead [1972], Mead and Hess [1973], and Hess et al. [1973] considered the effects that the Jovian satellites, in particular the moon Io, would have on the radially inward diffusing particles. They showed that unless the diffusion is rapid, few particles would be able to cross the moon's orbit. Practically all authors dealing with diffusion in Jupiter's magnetosphere have concluded that diffusion must be strong, in particular at small values of $L$ (Mcllwain parameter).

Data provided by Pioneer 10 and 11 have been analyzed to yield information about radial diffusion. Mogro-Campero [1976], Simpson et al. [1974], and Mogro-Campero and Fillius [1976], for example, use the observed decreases of particle fluxes at the orbits of the moons Amalthea, Io, and Europa to find diffusion coefficients. Their combined results seem to indicate an energy and $L$-dependent diffusion coefficient $D=$ $D_{0} L^{4}$ with $D_{0} \simeq 10^{-10}\left[R_{J}^{2} \mathrm{~s}^{-1}\right]$ for $\sim 5-\mathrm{MeV}$ electrons. $D_{0}$ increases with increasing energy. This is to be compared with the 'best' preencounter estimates of $D=2 \times 10^{-8} L^{2}\left[R_{J}{ }^{2} \mathrm{~s}^{-1}\right]$ by Birmingham et al. [1974] and $D=10^{-10} L^{3}\left[R_{J}^{2} \mathrm{~s}^{-1}\right]$ obtained by Coroniti [1974] on purely theoretical grounds. It seems that these are only marginally consistent with the results of Mogro-Campero and other observers. Thomsen and Goertz [1975] and Goertz [1976] have pointed out that the experimental estimates are based on rather crude and perhaps inconsistent assumptions and cannot necessarily be regarded as indicative of the true diffusion coefficients. Clearly, a second independent estimate of the diffusion coefficient is called for.

All authors assumed that no losses of particles occur in the inner Jovian magnetosphere other than those produced by the moons or by DIM radiation [Birmingham et al., 1974]. Coroniti [1974] assumes that losses due to whistler mode turbulence (pitch angle scattering) occur only outside $L=7$. There is, however, strong evidence that even inside $L=7$ whistler turbulence is present and that particles are precipitated into the loss cone. Scarf [1976], Scarf and Sanders [1976], Van Allen [1976], and Sentman and Van Allen [1976] show that the pitch angle distribution of electrons with $E>21 \mathrm{MeV}$ inside $L=7$ is compatible with whistler mode induced pitch angle diffusion.

\footnotetext{
${ }^{1}$ Now at George W. Downs Laboratory of Physics, California Institute of Technology, Pasadena, California 91125.

Copyright $(1976$ by the American Geophysical Union.
}

Thus loss-free diffusion is apparently not a good model. This conclusion was also reached by Fillius et al. [1976]. In this paper we show that a diffusion coefficient, considerably larger than any previous estimate, could be compatible with the observed phase space density variation if strong nonadiabatic losses are included.

It is clear that a treatment of the diffusion past the moons' $L$ shells, assuming no losses other than those induced by the moons, is only justified if the nonadiabatic losses due to pitch angle scattering are small. We show here that this may not be the case.

\section{ObSERVATIONS}

The University of Iowa instrument on Pioneer 10 had six distinct integral electron channels with adopted thresholds that allowed energy spectral analysis in the range of electron energy $50 \mathrm{keV} \lesssim E_{e} \lesssim 100 \mathrm{MeV}$. The University of lowa Pioneer 11 package had five electron channels which covered a similar range of energies with increased sensitivity at lower energies. The observations reported here will emphasize the near-equatorial data, and hence Pioneer 10 data will be stressed. The inbound Pioneer 11 data have been observed to be consistent with Pioneer 10. From absolute intensity measurements in the several integral energy channels, we specify a model electron differential energy spectrum which adequately describes the energy distribution for most of the Pioneer inbound and outbound passes.

Figure 1 shows typical data taken near the equatorial plane on the inbound Pioneer 10 pass. The spectrum is a hard power law in energy for low values of $E_{e}$ and becomes much steeper at high energies. The general form of the unidirectional differential spectrum is

$$
j=d J / d E=K E^{-15}(1+E / H)^{-n}
$$

For $40>r>20 R_{d}, H \sim 2-5 \mathrm{MeV}$, and the exponent $n$ ranges from $\sim 2$ to 3 . Similar spectra were observed at greater radial distances but with both $H$ and $n$ considerably larger. Figure 2 shows spectra taken inside $\sim 20 R_{J}$. $H$ and $n$ both appear to reach relative minima near $15 R_{J}$, and they increase strongly into $\sim 5 R_{J}$.

By using this form for the unidirectional intensity differential in energy and the observed values of $K, H$, and $n$, it is possible to compute the phase space density at constant first adiabatic invariant $\mu$. We compute the phase space density $f$ as a function of radial distance from the planet for near-equatorial mirroring electrons (second adiabatic invariant $I \approx 0$ ) from

$$
f(\mu)=j / p^{2}
$$

$p$ is the relativistic momentum of the particles. Examples of 


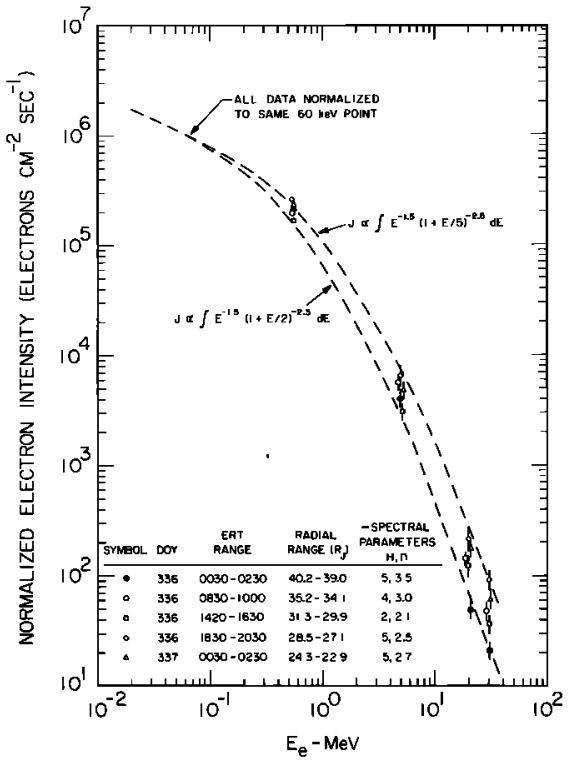

Fig. 1. Energy spectrum for electrons at $40 R_{J}>r>20 R_{J}$. The day of the year (DOY) refers to 1973 .

these profiles are shown in Figure 3 for $\mu$ values characteristic of the lower-energy electrons detectable by the University of Iowa instruments $(E<5 \mathrm{MeV})$ observed near the magnetopause boundary $\left(\mu_{1}=1.3 \times 10^{3} \mathrm{MeV} / \mathrm{G}, \mu_{2}=1.7 \times 10^{4}\right.$ $\left.\mathrm{MeV} / \mathrm{G}, \mu_{3}=5.9 \times 10^{5} \mathrm{MeV} / \mathrm{G}\right)$.

The profiles seem consistent with inward radial diffusion with the additional requirement of significant nonadiabatic losses in the regions near the plauet (inner magnetosphere). As discussed by Baker and Van Allen [1976], a possible explanation of both the shape of the observed energy spectrum and the huge decreases in phase space density inside $\sim 20 R_{J}$ consists of invoking pitch angle scattering due to whistler mode instability. Outside $\sim 20 R_{J}$ the phase space density profiles are rather inconclusive. Mcllwain and Fillius [1975] have derived similar phase space density profiles for electrons and have, likewise, concluded that local losses may be important. However, no analysis of loss rates and diffusion coefficients was made by either Baker and Van Allen or McIlwain and Fillius.

\section{INTER PRETATION}

We hypothesize that the observed phase space density profiles inside $\sim 20 R_{J}$ represent an equilibrium situation. The outer Jovian magnetosphere is undoubtedly highly time variable. We will hence concentrate on the inner magnetosphere. We seek solutions to the one-dimensional radial diffusion equation

$$
\partial f / \partial t=L^{2} \partial / \partial L\left(D / L^{2} \partial f / \partial L\right)-f / \tau=0
$$

The term $f / \tau$ is a sink term expressing losses with $\tau$ as a characteristic electron lifetime against nonadiabatic losses. A standard way of solving (3) is to assume

$$
D=D_{0} L^{n}
$$

and

$$
\tau=\tau_{0} L^{m}
$$

[see, e.g., Thorne, 1972]. The first assumption is reasonably
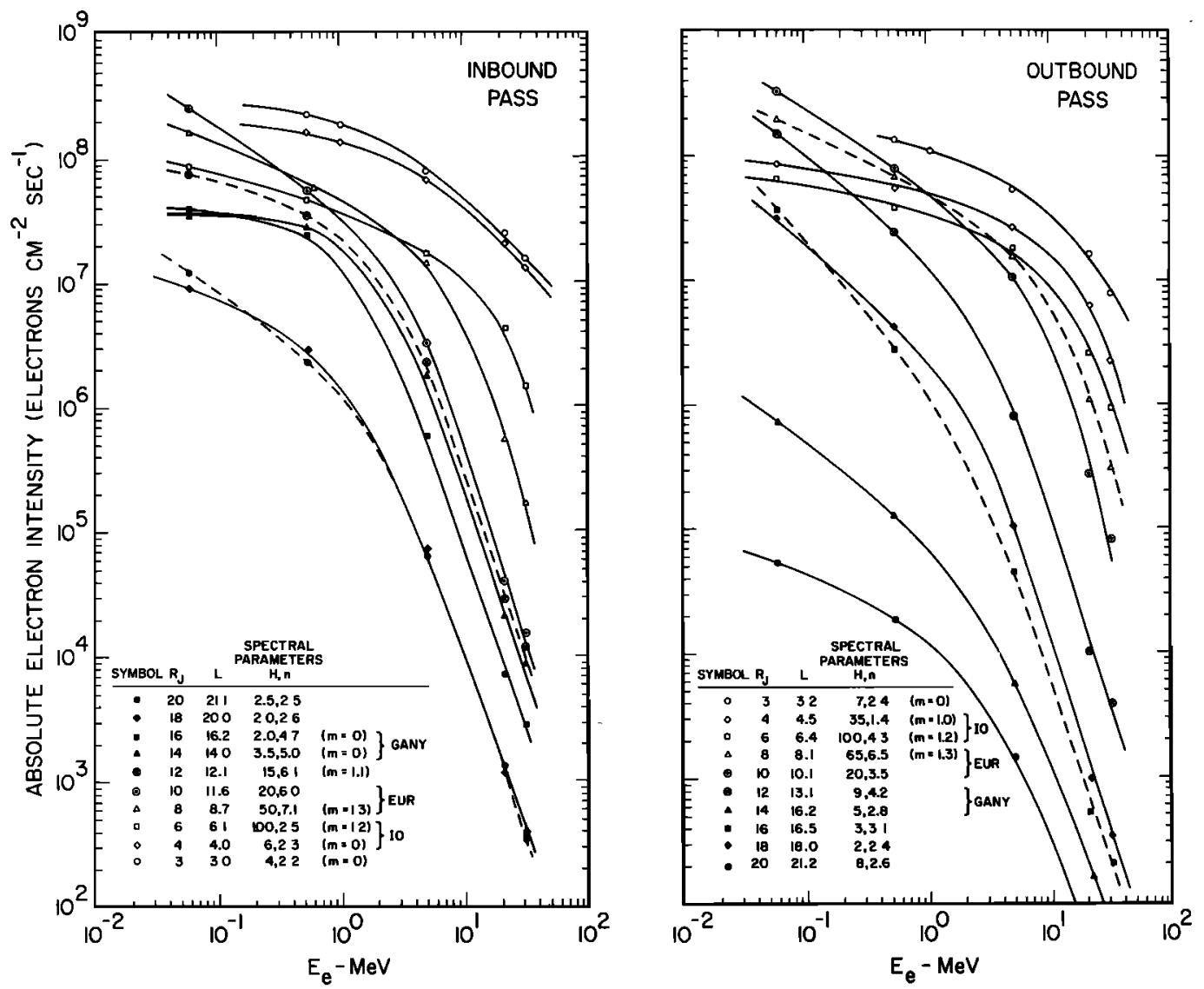

Fig. 2. Energy spectra for electrons inside $20 R_{J}$. 


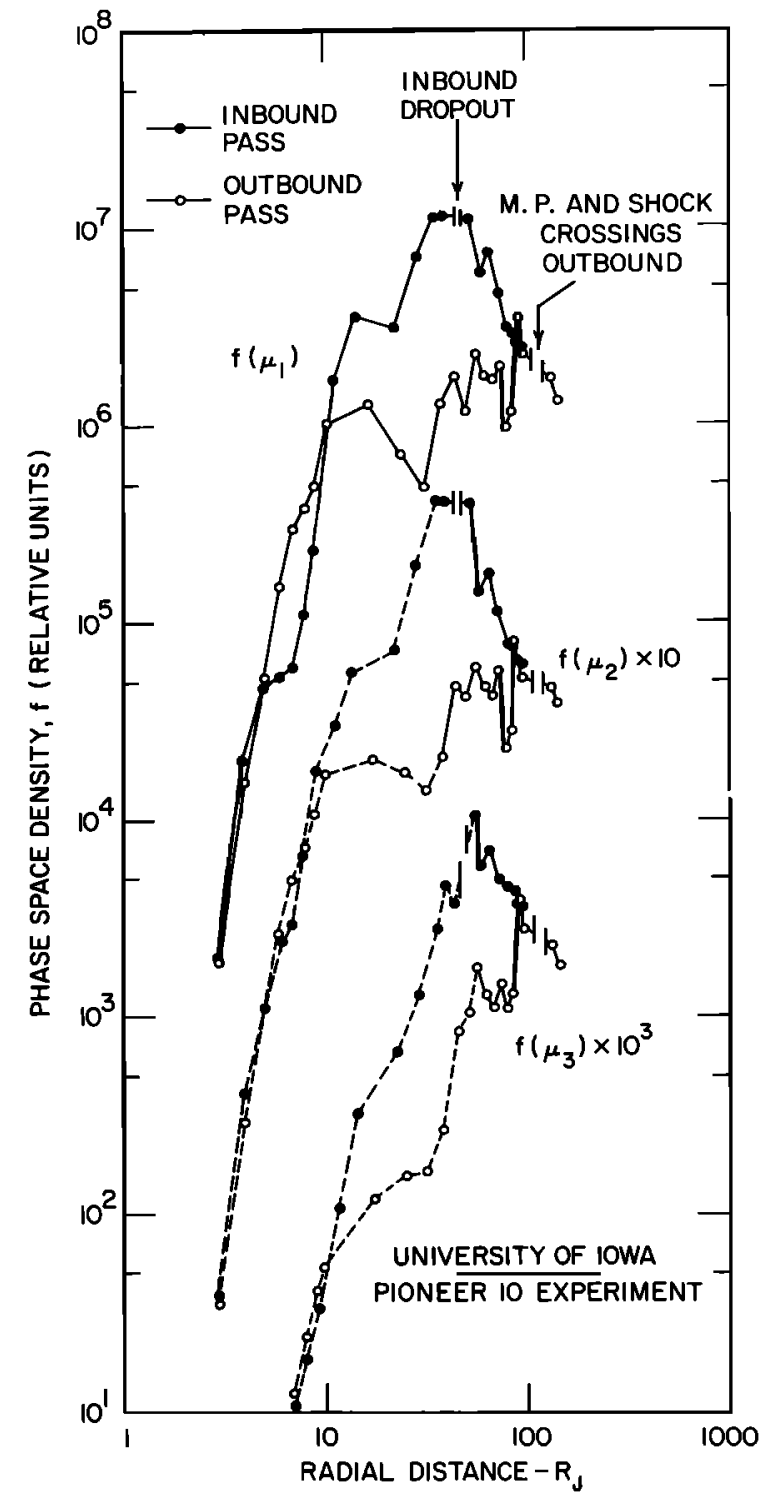

Fig. 3. Phase space densities calculated from the observed electron fluxes and spectra.

valid if diffusion is caused by magnetic field disturbances due to compressions of the magnetosphere $(n=10)$, by electric field disturbances due to changes of solar wind interaction with the magnetosphere $(n=6)$, or by electric field disturbances driven by large-scale turbulence in Jupiter's atmosphere $(n=2-3)$ [see, e.g., Mogro-Campero, 1976]. The second assumption may be valid if the losses are caused by strong pitch angle scattering as discussed by, e.g, Kennel and Petschek [1966]. For this case, $m \simeq 4$ and $\tau_{0}$ is the quarter-bounce period of the resonant particles $\left(\tau_{0} \leqslant 10 \mathrm{~s}\right)$. There is no direct evidence that the whistler mode induced pitch angle diffusion is either strong or weak. It seems that the pitch angle distributions of high-energy electrons $(E>21 \mathrm{MeV})$, i.e., those we are dealing with here, reported by Van Allen et al. [1975], Van Allen [1976], and Scarf and Sanders [1976] are more compatible with weak diffusion. However, this is not certain because no pitch angle measurements in the loss cone are available as yet. If, for example, radial diffusion would act as a strong source, even strong pitch angle diffusion would not necessarily imply an isotropic pitch angle distribution. The advantage of the assumption of strong pitch angle diffusion is the fact that quantitative estimates about the lifetime can be made. We try it here merely to show the effect that inclusion of strong losses has on the estimate of the diffusion constant. We are sure that losses occur but not whether they are of this form. In the earth's case, theoretical estimates for the lifetime of constant $\mu$ particles show $\tau$ to be nearly independent of $L$ [Thorne, 1972] at larger radial distances. Coroniti [1974] argues for a lifetime at Jupiter of the form $\tau=\tau_{0} L^{-1}$ assuming that $D=D_{0} L^{3}$ and that the integral fluxes of electrons with $E>0.1 \mathrm{MeV}$ are independent of $L$. There is evidence that the electron fuxes are constant only for $10<L<16$ [see, e.g., Van Allen, 1976]. It is clear that pitch angle scattering of electrons due to whistler mode turbulence will not be active outside $15 R_{J}$ where the electron's pitch angle distribution is dumbbell shaped [Sentman and Van Allen, 1976]. However, in this region the diffusion time which scales like $L^{2} / D \propto L^{2-n}$ is much smaller than the lifetime $\tau$, the term $f / \tau$ is small in comparison to the diffusion term, and an error in $\tau$ there is not very serious.

An analytic solution of (3) which goes to zero as $L \rightarrow 0$ with assumptions (4) and (5) is

$$
f \propto x^{a} K_{a}(x)
$$

where

$$
\begin{gathered}
x=\left(L / L_{0}\right)^{p} \quad a=(3-n) /[2-(m+n)] \\
p=[2-(m+n)] / 2
\end{gathered}
$$

$K_{\nu}(x)$ is the modified Bessel function of the second kind [Abramowitz and Stegun, 1964]. A similar solution was obtained by Thorne [1972]. The scale length $L_{0}$ is $L_{0}=\left[\tau_{0} D_{0} p^{2}\right]^{1 / 2 p}$. We have assumed that $p<0$, which is true for most values of $m$ and $n$ suggested above. It is obvious that if loss-free diffusion (i.e., $\tau_{0}$ $\rightarrow \infty$ ) would be the case, $f$ would have to be constant or $f \propto \ln$ $L$ for $n=3$. Clearly, this is not the case.

We have tried various values of $n$ and $m$ and have found that the best fit is obtained for $m=4, n=2$. Figure 4 shows the

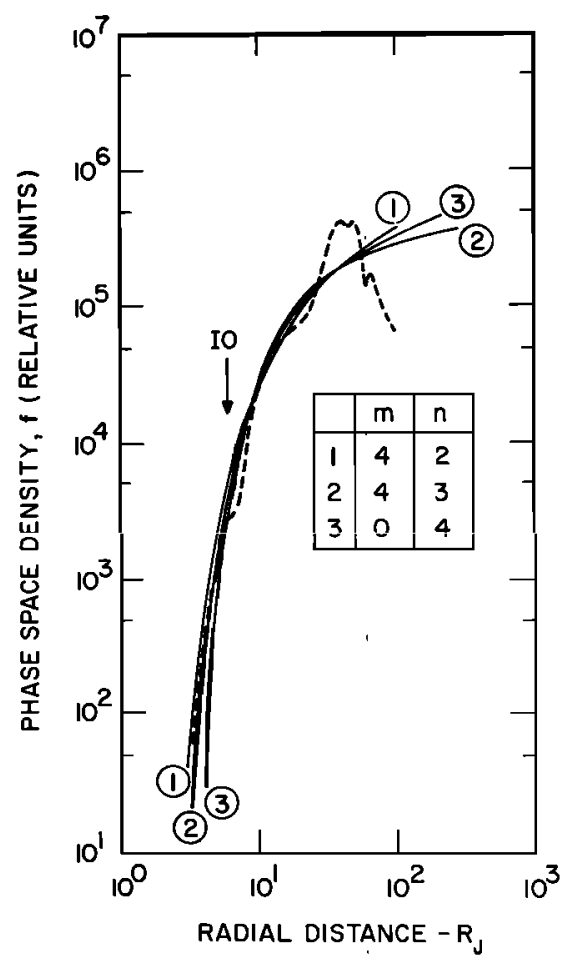

Fig. 4. Phase space density profiles for $\mu=1.7 \times 10^{4} \mathrm{MeV} / \mathrm{G}$. The dashed curves are analytic solutions with $D=D_{0} L^{n}$ and $\tau=\tau_{0} L^{m}$. 
TABLE 1. Values of $\tau_{0} D_{0}$ Obtained From Fits to Phase Space Density Profiles for Different Values of $m$ and $n$

\begin{tabular}{|c|c|c|c|c|c|}
\hline$m$ & $n$ & $p$ & $L_{0}$ & $\tau_{0} D_{0}, R_{J}^{2}$ & $\mu, \mathrm{MeV} / \mathrm{G}$ \\
\hline \multirow{2}{*}{4} & \multirow{2}{*}{2} & \multirow{2}{*}{-2} & 10 & $2.5 \times 10^{-5}$ & $1.3 \times 10^{3}$ \\
\hline & & & 7 & $1.0 \times 10^{-4}$ & $1.7 \times 10^{4}$ \\
\hline \multirow{2}{*}{4} & \multirow{2}{*}{3} & \multirow{2}{*}{$-\frac{5}{2}$} & 6 & $2.1 \times 10^{-5}$ & $1.3 \times 10^{3}$ \\
\hline & & & 6 & $2.1 \times 10^{-5}$ & $1.7 \times 10^{4}$ \\
\hline \multirow{2}{*}{2} & \multirow{2}{*}{3} & \multirow{2}{*}{$-\frac{3}{2}$} & 9.5 & $5.2 \times 10^{-4}$ & $1.3 \times 10^{3}$ \\
\hline & & & 8 & $8.7 \times 10^{-4}$ & $1.7 \times 10^{4}$ \\
\hline 0 & 4 & -1 & 25 & $1.6 \times 10^{-3}$ & $1.3 \times 10^{3}$ \\
\hline & 4 & -1 & 20 & $2.5 \times 10^{-3}$ & $1.7 \times 10^{4}$ \\
\hline
\end{tabular}

kind of fits obtained for the inbound Pioneer 10 phase space density profiles. It can be seen that the theoretical curves 2 and 3 tend to drop off too rapidly at small radial distances. Note that curve 3 differs from the observed curve by an order of magnitude inside $L=7$. We believe that this difference is statistically significant. Only curve 1 reproduces the decrease satisfactorily. Only for values of $4 \leq m+n \leq 7$ could a reasonable fit between the analytic solution (6) and the data be obtained. Thus $-5 / 2 \leq p \leq-1$. The values for $L_{0}$ range from 6 to 25 . Table 1 gives the results for various values of $m$ and $n$.

It is clear that without making assumptions about the lifetime we cannot estimate the diffusion coefficient. If, as indicated above, strong pitch angle diffusion is active, then $\tau_{0} \leqslant$ $10 \mathrm{~s}, m=4, n=2$, and $D \geq 2.5 \times 10^{-6} L^{2} R_{J}^{2} / \mathrm{s}$. This is several orders of magnitude larger than any previous estimate and should be regarded as an upper limit. It may be argued that a diffusion coefficient of this magnitude would not allow us to observe the absorption effect of the moons. However, we are dealing with particles that have an energy above $5 \mathrm{MeV}$ and above $17 \mathrm{MeV}$ at $L=6$. For these energies, absorption effects at the moons were observed to be small. Also it is not clear that by including the strong losses due to whistler mode interaction at the moons one would not preserve the absorption effects created by the moons for a longer time than was indicated by a loss-free diffusion model. Thus although loss rates and diffusion rates of this magnitude seem somewhat unrealistic, they cannot be ruled out, in particular, since $m=4$ and $n$ $=2$ provide a very good fit to the data.

We can use our method to estimate the loss rate if we adopt a value for the diffusion coefficient. The best fit to the data is obtained for $m+n=6$. Table 2 gives values for $\tau_{0}$ based on the diffusion coefficients of Birmingham et al. [1974], Coroniti [1974], and the 'observed' values of Mogro-Campero. We have also tried $n=4, m=0$ and find that in this case $L_{0}=25$. This case is also included in Table 2.
Although one cannot be sure that the losses are due to whistler turbulence, we will analyze our results in terms of whistler mode induced pitch angle diffusion. We do so because the pitch angle distribution of the electrons inside $L=10$ is also compatible with whistler mode induced pitch angle diffusion [Scarf, 1976; Scarf and Sanders, 1976].

Clearly, in all these cases, weak diffusion $\left(\tau \gg \tau_{\mathrm{m} ! n} \approx 10 L^{4}\right)$ prevails. Nevertheless, the loss rates are not trivial. The lifetimes can be related to the magnetic amplitude $\delta B$ of the whistler modes [see, e.g., Coroniti, 1974]

$$
\Omega_{-}(\delta B / B)^{2} \sim 1 / \tau
$$

where $\Omega_{-}$is the electron gyrofrequency and $B$ is the background field. Values for $\delta B / B$ are also indicated in Table 2 as well as the values for $\delta B$ at $L=10$ (assuming a dipole field with an equatorial field strength of $4 \mathrm{G}$ ). All estimates yield roughly the same values for $\delta B$ at $L=10$ and are consistent with estimates made previously by Coroniti [1974]. They should be detectable by future Jupiter probes. Finally, we would like to point out that the precipitated flux in all cases is quite significant at $L$ values beyond $L \approx 10$ in terms of possible heating of the Jovian ionosphere.

\section{Conclusion}

The variation of the phase space density for large $\mu$ electrons $\left(\mu>10^{3} \mathrm{MeV} / \mathrm{G}\right)$ is incompatible with loss-free radial diffusion. This and other evidence obtained from their pitch angle distributions suggest that these electrons are scattered into the loss cone by interaction with whistler mode turbulence. The magnitude of this scattering cannot be estimated from the data unless assumptions about the magnitude of the diffusion coefficient are made. It is remarkable that the data reported here are best fitted by assuming that the loss rate scales like $L^{-4}$ and the diffusion coefficient like $L^{2}$. A loss rate which scales like $L^{-4}$ could indicate that the pitch angle scattering due to whistlers is strong. If that is the case, the diffusion coefficient must be large. Using previous estimates for the diffusion coefficients, we find that the data are also compatible with weak pitch angle scattering only. Presently, available data do not allow an unambiguous decision as to whether pitch angle diffusion is strong or weak. It is thus essential that future Jupiter probes measure the amplitudes of the whistlers which almost certainly exist in the inner Jovian magnetosphere.

Acknowledgments. It is a pleasure to acknowledge helpful discussions with J. A. Van Allen, M. F. Thomsen, and D. D. Sentman. Thrs work was supported in part by NSF grant GA-31676 and NASA contracts NAS2-5603 and NAS2-6553.

TABLE 2. Values of Loss Times $\tau_{0}\left(\tau_{0}=\tau_{0} L^{m}\right)$ Based on Previously Published Diffusion Coefficients

\begin{tabular}{|c|c|c|c|c|c|c|}
\hline$m$ & $n$ & $\tau_{0}, \mathrm{~s}^{*}$ & $\tau_{0}, \mathbf{s} \dagger$ & $\tau_{0,}, s \neq$ & $(\delta B / B)$ & $\delta B, \mathrm{~m} \gamma \S$ \\
\hline \multirow{2}{*}{4} & 2 & $1.25 \times 10^{4}$ & & & $1.06 \times 10^{-0} \times L^{-1 / 2}$ & 0.13 \\
\hline & 2 & $5 \times 10^{4}$ & & & $5.3 \times 10^{-7} \times L^{-1 / 2}$ & 0.07 \\
\hline \multirow{2}{*}{4} & 3 & & $2.1 \times 10^{5}$ & & $2.6 \times 10^{-7} \times L^{-1 / 2}$ & 0.03 \\
\hline & 3 & & $2.1 \times 10^{5}$ & & $2.6 \times 10^{-7} \times L^{-1 / 2}$ & 0.03 \\
\hline \multirow{2}{*}{2} & 3 & & $5.2 \times 10^{6}$ & & $5.2 \times 10^{-8} \times L^{1 / 2}$ & 0.07 \\
\hline & & & $8.7 \times 10^{8}$ & & $4.0 \times 10^{-8} \times L^{1 / 2}$ & 0.05 \\
\hline \multirow{2}{*}{0} & 4 & & & $1.6 \times 10^{7}$ & $3.0 \times 10^{-8} \times L^{3 / 2}$ & 0.38 \\
\hline & 4 & & & $2.5 \times 10^{7}$ & $2.4 \times 10^{-8} \times L^{3 / 2}$ & 0.30 \\
\hline
\end{tabular}

${ }^{*} D=2 \times 10^{-8} L^{2}$.

$+D=10^{-10} L^{3}$

$\ddagger D=10^{-10} L^{4}$.

§At $L=10$. 
The Editor thanks 'two anonymous referees' for their assıstance in evaluating this report.

\section{REFERENCES}

Abramowitz, M., and I. A. Stegun, Handbook of Mathematical Functions, Appl. Math Ser., vol. 55, National Bureau of Standards, Washington, D. C., 1964.

Baker, D. N., and J. A. Van Allen, Energetic electrons in the Jovian magnetosphere, J. Geophys. Res., 81(4), 617-632, 1976.

Birmingham, T., W. Hess, T. Northrop, R. Baxter, and M. Lojko, The electron diffusion coefficient in Jupiter's magnetosphere, J. Geophys. Res., 79, 87-97, 1974.

Coroniti, F. V., Energetic electrons in Jupiter's magnetosphere, $A s$ trophys. J., 27, 261-281, 1974.

Fillius, R. W., C. E. Mcllwain, A. Mogro-Campero, and G. Steinberg, Evidence that pitch angle scatterıng is an important loss mechanism for energetic electrons in the inner radiation belt of Jupiter, Geophys. Res. Lett., 3, 33-36, 1976.

Goertz, C. K., Jupiter's magnetosphere: Particles and fields, in Jupiter. the Giant Planet, edited by T. Gehrels, University of Arizona Press, Tucson, in press, 1976.

Hess, W. N., T. J. Birmingham, and G. D. Mead, Jupiter's radiation belts: Can Pioneer 10 survive?, Science, 182, 1021-1022, 1973.

Kennel, C. F., and H. E. Petschek, Limil on stably trapped particle fluxes, J. Geophys. Res., 7I, 1-28, 1966.

Mead, G. D., The effect of Jupiter's satellites on the diffusion of protons, in Proceedings of the Jupiter radiation Belt Workshop. Tech. Mem. 33-543, edited by A. J. Beck, pp. 271-276, Jet Propulsion Laboratory, Pasadena, California, 1972

Mead, G. D., and W. N. Hess, Jupiter's radiation belts and the sweeping effect of its satellites, J. Geophys. Res., 78, 2793-2811, 1973.

Mcllwain, C. E., and R. W. Fillius, Diflerential spectra and phase space densitıes of trapped electrons at Jupiter, J. Geophys. Res., 80, 1341-1345, 1975.

Mogro-Campero, A., Absorption of radiation belt particles by the inner satellites of Jupiter, in Jupiter, the Giant Planet, edited by $\mathrm{T}$ Gehrels, University of Arizona Press, Tucson, in press, 1976.

Mogro-Campero, A., and W. Fillius, The absorption of trapped particles by the inner satellites of Jupiter and the radial diffusion coefficient of particle transport, J. Geophys. Res., 8I(7), 1289-1295, 1976.

Scarf, F. L., Plasma physics and wave-particle interaction at Jupiter, in Jupiter, the Giant Planet, edited by T. Gehrels, University of Arizona Press, Tucson, in press, 1976.

Scarf, F. L., and N. L. Sanders, Some comments on the whistler mode Instability at Jupiter, J. Geophys. Res., 81(10), 1787-1790, 1976.

Sentman, D. D., and J. A. Van Allen, Angular distributions of electrons of energy $E_{e}>0.06 \mathrm{MeV}$ in the Jovian magnetosphere, $J$. Geophys. Res., 8I(7), 1350-1360, 1976.

Simpson, J. A., D. Hamilton, G. Lentz, R. B. McKibben, A. MogroCampero, M. Perkins, K. R. Pyle, A. J. Tuzzolino, and J. J. O'Gallagher, Protons and electrons in Jupiter's magnetic field: Results from the University of Chicago experiment on Pioneer 10 , Science, 183, 306-309, 1974.

Thomsen, M. F., and C. K. Goertz, Satellite sweep-up effects at Jupiter (abstract), Eos Trans. AGU, 56, 428-429, 1975.

Thorne, R. M., The importance of wave particle interactions in the magnetosphere, in Critical Problems of Magnetospheric Physics, edited by E. R. Dyer, pp. 211-226, National Academy of Sciences, Washington, D. C., 1972.

Van Allen, J. A., High-energy particles in the Jovian magnetosphere, in Jupiter, the Giant Planet, edited by T. Gehrels, University of Arizona Press, Tucson, in press, 1976.

Van Allen, J. A., B. A. Randall, D. N. Baker, C. K. Goertz, D. D. Sentman, M. F. Thomsen, and H. R. Flindt, Pioneer 11 observations of energetic particles in the Jovian magnetosphere, Science, $188,459-462,1975$.

(Received January 12, 1976; accepted May 26, 1976.) 